\title{
Successes and Challenges of Implementing Teleprehabilitation for Onco- Surgical Candidates and Patients' Experience: A Retrospective Pilot-Cohort Study
}

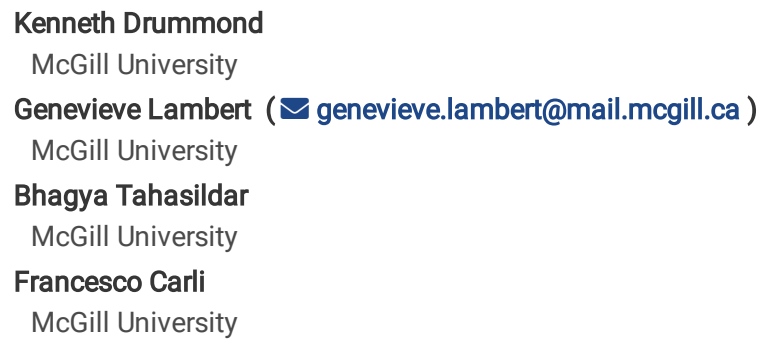

Research Article

Keywords: Prehabilitation, Telehealth, Functional Capacity, Teleprehabilitation, Surgical Oncology, Pandemic, Exercise, Nutrition, Behavioural counseling.

Posted Date: November 12th, 2021

DOI: https://doi.org/10.21203/rs.3.rs-1021190/v1

License: @) (1) This work is licensed under a Creative Commons Attribution 4.0 International License. Read Full License 


\section{Abstract}

Purpose: This study aimed to document the successes and challenges of teleprehabilitation programs for cancer patients undergoing surgery.

Method: This pilot-cohort study included adults scheduled for elective cancer surgery, referred to the prehabilitation clinic to engage in physical activity and received a teleprehabilitation program between August 1st 2020 and February 28th 2021. Using a technology platform that included a tablet and was wearable, data were acquired through virtual physical activity monitoring in addition to patient charts.

Results: Ten patients (8 males and 2 females; mean age: 68.3 years, SD: 11.96) diagnosed with various thoraco-abdominal malignancies were included in the current descriptive study. The successes identified were related to recruitment and assessment, improvement in functional capacity, clinic scheduling and interventions, and optimal medical follow-up. The challenges identified were related to the adoption of the technologies by patients and the multidisciplinary team, the accurate acquisition of patient physical activity data, and the initial costs to acquire the new technologies. Patients were satisfied with the teleprehabilitation program (i.e., services delivered; average appreciation: $96 \%$ ), and they perceived the technologies provided to be $90 \%$ userfriendly.

Conclusion: The findings of the current study are paramount in view of the current international health paradigm changes prioritizing remote interventions facilitated through digital communication technologies. It provides important insight into the clinical application of telehealth in elderly populations, notably in the context of acute preoperative cancer care. This article may provide guidance for other cancer care facilities aiming to implement teleprehabilitation programs.

\section{Background}

The COVID-19 pandemic has had an immense impact on public health, challenging healthcare systems and institutions to adapt to global circumstances and uphold the same quality of care for patients. To this end, many surgeries and medical procedures were delayed indefinitely, prioritizing cases that required urgent interventions, thus creating a backlog of approximately two years of cancer surgery (1-3). In addition, the risk of viral exposure is a serious concern for these patients, as they are often immunocompromised and have significant comorbidities and poor lifestyle habits. The implications of preoperative harmful lifestyle modifications, isolation and quarantine may increase perioperative morbidity, postoperative recovery and mortality (4-6).

Prehabilitation aims to improve postoperative outcomes by addressing modifiable risk factors. It has been increasingly recognized to improve functional and clinical trajectories, thereby reducing the burden on both patients and the healthcare system (7). Multimodal prehabilitation interventions are multidisciplinary and typically include an exercise training program, nutritional optimization, psychosocial counseling, pharmacological optimization, glycemic control, and smoking cessation if needed (8).

In view of the pandemic, remote delivery of prehabilitation services has been suggested (9) using telehealth platforms paired with technologies (i.e., teleprehabilitation) to address concerns related to cancer patients' risks, isolation, and deterioration of their health status.

This pilot-cohort study aims to document 1) the success and challenges associated with the delivery of a teleprehabilitation program to cancer patients and 2) the patients' experience of the program.

\section{Methods}

\section{Study Design, Ethical Approval \& Patient Selection}

This retrospective pilot-cohort study describes the delivery of teleprehabilitation programs to cancer patients during the COVID-19 pandemic from August 2020 until February 2021. It was approved by the ethical review board of the McGill University Health Centers (MUHC; Study ID: 2021-7666, approval granted on March 30th, 2021). All methods were carried out in accordance with relevant guidelines and regulations. The inclusion criteria for this cohort study were adults scheduled for elective thoracic and abdominal tumor resection. All patients had a good comprehension of the English or French language and agreed to participate in this study by signing a consent form.

\section{Referral}

Following referral by the MUHC surgeons to the prehabilitation clinic, patients were contacted and offered technology-assisted prehabilitation. Those who accepted were scheduled for an initial assessment at the clinic.

\section{Clinical Evaluation Process}

Baseline and subsequent evaluations were conducted in the prehabilitation clinic, as permitted by recommendations from the Public Health Authorities and Emergency Measures Coordination Center of the MUHC. The baseline evaluation was conducted by a physician, an exercise physiologist, a registered dietician and, if needed, a psychology-trained nurse. The evaluations lasted approximately one hour, during which clinical status and functional capacity were assessed with a battery of standardized tests as previously described $(8,14)$. Patients also met in person or virtually with a dietitian for nutrition counseling and a nurse specializing in psychosocial counseling. The patients then received a personalized multimodal prehabilitation program. The trajectory is represented in Figure 1. To limit unnecessary additional hospital visits, prehabilitation evaluations (baseline, 24 hours prior to surgery, and 4 and 8 weeks after surgery) were timed to coincide with patients' other essential medical appointments.

\section{Technologies and Material}


Patients attended a 45-minute technical workshop on the different applications and technologies $(10,11)$ They were also provided with a training watch paired with either a numerically coded email (generated by the clinic; $n=8)$ or alternatively their personal email $(n=2)$. The emails were used to register the technologies with the coded accounts for Polar Flow and Zoom applications. As the latter applications were core to the teleprehabilitation program, the clinic furnished tablets, with the predownloaded applications to facilitate its delivery. The tablets were also equipped with premade educational videos created by the prehabilitation clinic team on nutrition optimization (healthy eating, improving protein and energy intake, portion size, glycemic control) and relaxation exercises (breathing exercise, relaxation, imaging, visualization). Tablets were loaned to seven patients, while three patients were equipped with their personal devices. Beyond the watch and tablet, one patient was also provided with a magnetically braked upright cycle ergometer, which was lent to him for aerobic exercise due to concerns pertaining to knee pain and climate concerns. All patients received complementary material during the initial visit at the prehabilitation clinic: nutritional supplements of whey protein, relaxation recordings, elastic bands (Theraband $\circledast$ ), a threshold inspiratory muscle trainer (IMT) device, and a booklet with a personalized exercise prescription.

\section{Description of The Data Retrieved}

All outcomes were captured via data collected from the Polar Flow application, self-report questionnaires, and patient chart review. Data collected from the Polar Flow application were as follows: the weekly minutes recorded as exercise, the daily steps, the frequency and duration of resistance and the number of aerobic sessions. Self-report questionnaires included a mental well-being questionnaire (Hospital Anxiety and Depression Scale; HADS (20)) and a postintervention satisfaction questionnaire (Appendix A). Outcomes obtained from patient charts included neoadjuvant therapies (NAT), emergency department (ED) visits, surgical procedure, length of hospital stay (LOHS), and intra- and postoperative complications.

\section{MUHC Standard of Care During Pandemic}

Following the onset of the global COVID-19 pandemic in March 2020, hospital access and functions were restricted, and the standards of care and services provided to surgical candidates were negatively affected. Surgical patients were able to be seen in person by nurses, internists and anesthesiologists in the preoperative clinic. Patients were also asked to perform a precautionary COVID test 24-48 hours preceding their surgery to confirm that they were not positive carriers of the virus.

\section{Outcomes}

The primary outcome was the qualitative description of the patient's perioperative trajectory of care, including the completion rate, intervention-related adverse events, drop-outs and exercise metrics, preoperative functional and clinical trajectories, and programme successes and challenges.

The secondary outcome was the patients' experience with the program presented with qualitative analysis related to the advantages and disadvantages of technology support. The quotes were taken from the satisfaction questionnaires and from the exercise physiologists' clinical notes. The quotes were reported in an anonymized manner relating to two main themes: their satisfaction with the services and their experience with the technologies (12,13).

\section{Data analysis}

Descriptive analyses were used for demographics, program exercise metrics, baseline and preoperative functional and clinical outcomes. Means and standard deviations were reported when data were normally distributed; alternatively, medians and ranges were used for parameters with skewed distributions. The distribution of the data was first visually appraised with respect to the interquartile ranges, and in cases of uncertainty, the Shapiro-Wilk test was used to confirm normality (16).

Additional statistical analyses include a paired t-test comparing means from pre- (T0) and posttests (T1) performed to assess changes in functional performance and exercise metrics (first week compared to last week of intervention).

The abovementioned calculations were performed using PASW Statistics software version 24.0, with confidence intervals and significance levels preset at $95 \%$ and 0.05 , respectively. (SPSS Inc, Chicago, Illinois).

\section{Results}

\section{Patients' Characteristics}

Ten patients received teleprehabilitation services. On average, patients lived $33.75 \mathrm{~km}$ (range: $2.7 \mathrm{~km}$ to $193.5 \mathrm{~km}$; one-way trip) from the MGH. Details of the cohort's characteristics can be found in Table 1. 
Table 1

Demographic and Health Characteristics

\begin{tabular}{|c|c|c|}
\hline & Cohort & Missing Data \\
\hline Age, years & $68[52-88]$ & \\
\hline Sex-male, n (\%) & $8(80 \%)$ & \\
\hline Weight, kg & $75.4(7.4)$ & \\
\hline BMI, kg/m² & $25.5[22.6-31.1]$ & \\
\hline \multicolumn{3}{|l|}{ Cancer Type, n (\%) } \\
\hline Colorectal & 2 & \\
\hline Lung & 3 & \\
\hline Retroperitoneal Sarcoma & 3 & \\
\hline Esophagogastric & 2 & \\
\hline \multicolumn{3}{|l|}{ Neoadjuvant Therapies, $n$} \\
\hline Chemotherapy & 3 & \\
\hline Radiotherapy & 3 & \\
\hline Chemo-radiotherapy & 1 & \\
\hline PG-SGA, score & $7(1-16)$ & 2 \\
\hline Alcohol consumer, $n$ & 6 & \\
\hline Current smoker, n & 7 & \\
\hline HADS -Anxiety & $3(1-14$ & 1 \\
\hline HADS -Depression & $4(1-11)$ & 1 \\
\hline \multicolumn{3}{|l|}{ Blood Biochemistry } \\
\hline C-reactive protein, $\mathrm{mg} / \mathrm{L}$ & $7.3(1.67-130)$ & 1 \\
\hline Albumin, g/L & $40.7(2.4)$ & 1 \\
\hline $\mathrm{HbA} 1 \mathrm{c}, \%$ & $6.16(0.5)$ & 3 \\
\hline Hemoglobin, g/L & $130.2(22.3)$ & - \\
\hline BNP & $38(15-368)$ & 2 \\
\hline
\end{tabular}

The most common comorbidities were hypertension $(n=8)$, arthritis $(n=4)$, cardiovascular disease $(n=4)$, dyslipidemia $(n=3)$, diabetes $(n=3)$, chronic obstructive pulmonary disease $(n=3)$, and obstructive sleep apnea $(n=2)$. Other medical conditions reported in the cohort included Guillain-Barré syndrome $(n=1)$, gastroesophageal reflux disease $(n=1)$, depression $(n=1)$, spinal stenosis $(n=1)$, chronic kidney disease $(n=1)$, asthma, tinnitus, mild hearing loss and kidney stones $(n=1)$.

\section{The Program: Functional and clinical trajectories, Success \& Challenges Teleprehabilitation Program metrics}

The median teleprehabilitation period was 9.5 weeks (IQR 2 - 15) from baseline evaluation and continued throughout neoadjuvant therapies in 9 patients until surgery. One patient did not undergo surgery due to disease progression. No intervention-related adverse events were reported. 
Table 2

Exercise Metrics

\begin{tabular}{|c|c|c|c|c|c|c|c|c|c|c|c|}
\hline \multirow[t]{2}{*}{ ID } & \multirow[t]{2}{*}{$\begin{array}{l}\text { Program } \\
\text { Duration }\end{array}$} & \multicolumn{2}{|c|}{$\begin{array}{l}\text { Daily Step } \\
\text { Count }\end{array}$} & \multicolumn{2}{|c|}{$\begin{array}{l}\text { Weekly } \\
\text { Exercise [min] }\end{array}$} & \multicolumn{2}{|c|}{ Resistance Exercise } & \multicolumn{2}{|c|}{ Aerobic Exercise } & \multicolumn{2}{|c|}{$\begin{array}{l}\text { Exercise Counseling } \\
\text { Session }\end{array}$} \\
\hline & & $\begin{array}{l}1 \text { st } \\
\text { Week }\end{array}$ & $\begin{array}{l}\text { Last } \\
\text { Week }\end{array}$ & $\begin{array}{l}1 \text { st } \\
\text { Week }\end{array}$ & $\begin{array}{l}\text { Last } \\
\text { Week }\end{array}$ & Session/Wk & Duration/Session & Session/Wk & Duration/Session & Total & Duration/Session \\
\hline 1 & 13 & 6427 & 6453 & 65 & 103 & 1.08 & 32 & 1.85 & 115 & 20 & 27 \\
\hline 2 & 5 & 5977 & 7455 & 194 & 136 & 2.4 & 41 & 5.4 & 36.5 & 14 & 21 \\
\hline 3 & 2 & 7480 & 6303 & 193 & 107 & 2 & 17.5 & 4.5 & 17.5 & 2 & 18 \\
\hline 4 & 11 & 4600 & 7137 & 30 & 451 & 2.91 & 32 & 5.55 & 53 & 9 & 34 \\
\hline 5 & 6 & 2946 & 4100 & 152 & 229 & 2 & 24 & 3.83 & 23 & 12 & 36 \\
\hline 6 & 12 & 4693 & 5994 & 187 & 221 & 0.83 & 30 & 4.17 & 55 & 12 & 27 \\
\hline 7 & 15 & 1161 & 4500 & 244 & 355 & 2.73 & 32.5 & 4.4 & 33 & 14 & 37 \\
\hline 8 & 8 & 4821 & 2770 & 84 & 169 & 2.13 & 43 & 3.125 & 50 & 8 & 60 \\
\hline 9 & 13 & 5000 & 9760 & 60 & 200 & 0.69 & 37.5 & 1 & 32 & 10 & 36 \\
\hline 10 & 10 & 5845 & 4651 & 100 & 120 & 1.5 & 30 & 1.4 & 30 & 16 & 60 \\
\hline
\end{tabular}

ID: patient identifier, 1 to 10; Program Duration: unit = weeks; Weekly Exercise: data recorded voluntarily as exercise by patient on the polar watch, unit = minutes; Daily Step Count: data recorded by the polar watch, unit: mean step/day; Resistance Exercise and Aerobic Exercise: data recorded voluntarily as exercise by patient on the polar watch. 1st week. data collected during the patient's first week of the program; Last week. data collected during the patient's last week before their surgery; Session/wk. mean number of sessions per week; Duration/session: mean duration per session; unit: minutes; Total: total number of sessions through the program supervised by the exercise physiologist.

During the first week, the average daily step count was 4895 (SD: 1795) and increased by 1358 steps (SD: 1989) by the last week of prehabilitation (P-value $<0.001$ ), with six out of ten patients increasing their daily step count. (Table 2)

Although the median weekly time exercising was not significantly different from the Canadian recommendation of at least 150 minutes of moderate- to high-intensity exercise per week, the duration of exercise between the first and last weeks of prehabilitation increased by 58 minutes (range: -86 to 421 minutes; P-value: 0.093), with eight out of ten patients increasing their weekly time spent exercising.

\section{Preoperative Functional Capacities}

During the preoperative period, no significant increase in 6 MWD was shown, except for TUG and STS (P-value: 0.017 and P-value: 0.002 , respectively; Table 3).

Table 3

Preoperative Change in Functional Capacities

\begin{tabular}{|c|c|c|c|c|c|}
\hline & $\mathrm{n}$ & Baseline & $\mathrm{n}$ & Preoperative & P-value \\
\hline \multicolumn{6}{|l|}{ Six-minute walking distance } \\
\hline Actual, meters ${ }^{b}$ & 9 & $426[210-660]$ & 9 & $487[230-603]$ & 0.208 \\
\hline Timed-up and go, seconds ${ }^{b}$ & 9 & $7.87[5.47-8.48]$ & 8 & $5.58[4.23-8.35]$ & 0.017 \\
\hline Sit-to-Stand, repetitions ${ }^{a}$ & 10 & $11.5(6.19)$ & 9 & $12.78(6.03)$ & 0.002 \\
\hline \multicolumn{6}{|l|}{ Arm-Curl Test, repetitions ${ }^{a}$} \\
\hline Right & 8 & $18(5.81)$ & 8 & $18.88(2.90)$ & 0.186 \\
\hline Left & 8 & $17.88(6.90)$ & 8 & $18.5(2.78)$ & 0.074 \\
\hline \multicolumn{6}{|l|}{ Hand-Grip Strength, kg ${ }^{\text {b }}$} \\
\hline Right & 10 & $30[10-46]$ & 8 & $30[12-52]$ & 0.085 \\
\hline Left & 10 & $30[10-38]$ & 8 & $30[12-42]$ & 0.394 \\
\hline
\end{tabular}

\section{Clinical Trajectory}


Postoperative recovery was uncomplicated for most patients, with serious complications reported in only three patients (CCl scores: $100,33.5$ and 62.2 ). One patient was readmitted for drainage of a pelvic abscess. There was one death. (Table 4).

Table 4

Surgical and Post-Operative Outcomes

\begin{tabular}{|l|l|}
\hline Surgery & \\
\hline Surgical Approach & 4 \\
Open & 5 \\
Minimally invasive & \\
\hline Surgical Procedure & 2 \\
Lobectomy & 2 \\
Esophagectomy & 3 \\
Retroperitoneal resection & 2 \\
\hline Colectomy & \\
\hline Post-Operative Recovery \& Morbidity & 5 \\
\hline Patients without complications & 6 [2 - 47] \\
\hline LOHS & 1 \\
\hline Readmissions & 1 \\
\hline Reoperations & 1 \\
\hline In-hospital mortality & \\
\hline Data are presented as n: absolute number of patients, or median [range]. \\
\hline
\end{tabular}

Program's Successes

1. Recruitment and assessment: None of the patients opposed receiving technology-supported prehabilitation interventions. The initial evaluation was either performed at the prehabilitation clinic $(n=7)$, at the surgeon's office $(n=2)$, or while hospitalized $(n=1)$.

2. Clinical scheduling \& interventions: In the context of the pandemic, evaluations by the exercise physiologist and the dietitian in the clinic were limited to one patient per hour separated by 15-minute sanitation breaks. Remote consultations did not impose a burden on in-person clinical functions. The training watches allowed patients to record and self-monitor their training sessions. The exercise physiologists were able to asynchronously monitor patients' patterns of physical activity, pertinent accelerometric, and biometric data objectively. The Zoom application allowed the exercise physiologists to virtually meet with patients, visualize and adapt exercises to the patient's environment while synchronously providing corrective cues and appropriate health counseling.

3. Optimal medical follow-up: All patients were contacted by the exercise physiologist through the Zoom interface at least once per week to revise their weekly physical activity levels, assess patient progress, review the exercises provided and modify the prescription as needed. The virtual exercise counseling sessions provided exercise physiologists with the opportunity to inquire about health, symptoms and general well-being, which was communicated with the multidisciplinary team for the delivery of additional medical $(n=8)$, nutritional $(n=7)$, and psychosocial $(n=6)$ support over phone calls.

Program's Challenges

1. Patient learning curve: Seven patients used the tablets, while all used the polar training watch. Most patients required assistance from a family member $(n=4)$, caregiver $(n=1)$ or exercise physiologists $(n=3)$ to assist them in using the technologies and applications. To this end, 4 patients required continuous assistance throughout the preoperative period, two others required aid only on one occasion (during the first 7 to 14 days), and the rest did not require any assistance. The three patients who required the exercise physiologist's help were due to a) challenges in downloading the applications on their personal devices ( $n=1)$ and $b)$ performing a factory reset of the device $(n=2)$.

2. Physical activity data acquisition: Although patients were trained to actively begin and terminate the recordings and synchronize the watch with the Polar Flow application, patients tended to forget, thus resulting in missed collection of heart rate during the exercise session. All patients, except one, wore the watch regularly ( $>4$ days/week).

3. Multidisciplinary team adjustment to new technologies: Only the exercise physiologists and research coordinator communicated with the patient using the Zoom application. Patients received an initial nutritional consultation with a registered dietician either in person $(n=8)$ or over the phone ( $n=3)$, and all patients received psychosocial counseling sessions over the phone $(n=8)$, except two who were already being supported by other psychology-trained personnel prior to their recruitment.

4. Postoperative loss to follow-up: Half of the cohort's patients were lost to follow-up. This was in part due to disease progression ( $n=1)$, the commencement of adjuvant therapy $(n=1)$, postoperative complications $(n=1)$, mortality $(n=1)$, and discontinued interest $(n=1)$. 
5. Cost: Patients were lent technologies (Polar Ignite watch, $n=10$ and a Samsung Galaxy tablet, $n=7$ ) that facilitate remote real-time counseling and physical activity monitoring. The cost of the tablet was approximately $\$ 350$, while that of the watch was $\$ 250$, with an average of $\$ 15$ to $\$ 20$ per month for patients who required internet data $(n=2)$. Since the tablets and the watches were reusable, patients were asked to return at the end of the study.

\section{Patients Self-Report Experience}

The Program

Eight patients were able to complete a satisfaction questionnaire pertaining to their participation in the program, whereas two patients were unable to complete the questionnaire because of surgical complications $(n=1)$ and mortality $(n=1)$. The median satisfaction score for teleprehabilitation was $96 \%$ (range: 83 to $100 \%$ ). The average perceived usefulness score of prehabilitation services was $88 \%$ (SD: 10.43). Patients perceived benefits of receiving teleprehabilitation on their physical fitness $(n=8)$, mental health $(n=6)$, symptoms of their condition (e.g., pain, nausea, fatigue, etc.; $n=3)$, social health ( $n=2)$, and diet $(n=1)$. Other benefits mentioned included "General wellness and support" [Patient \#4, \#5]. Moreover, one patient attributed her early discharge to her perceived improvements in physical fitness. Quotes from patients are presented in Table 5.

Table 5

Quotes from Patients

\begin{tabular}{|c|c|c|}
\hline Theme & $\mathbf{n}$ & Quotes \\
\hline \multirow[t]{3}{*}{ Support } & 9 & $\begin{array}{l}\text { "I would not have been able to endure the treatments and the surgery thereafter had it not been for the continuous } \\
\text { support I was receiving through the digital platform" [Patient\#4] }\end{array}$ \\
\hline & & $\begin{array}{l}\text { - "I felt there was somebody on the other side [of the application], the team was reliable [...] The exercise physiologist } \\
\text { was professional and knowledgeable, and I wouldn't have completed the program without them" [Patient\#1] }\end{array}$ \\
\hline & & - "My son was able to do [the aerobic exercise] with me" [Patient\#5] \\
\hline \multirow{2}{*}{$\begin{array}{l}\text { Inability to enrol in the } \\
\text { program if in-person } \\
\text { interventions }\end{array}$} & 5 & $\begin{array}{l}\text { - "It takes the tablet. I do not think I could have come [at prehabilitation clinic for the interventions], with the medical } \\
\text { appointments and everything" [Patient\#5] }\end{array}$ \\
\hline & & • "It would have been impossible due to how far away I live from the hospital" [Patient\#2] \\
\hline Technological literacy & 2 & $\begin{array}{l}\text { "As you know, I have never used these technologies before. I do not own a phone, so it took me some time to learn } \\
\text { how to use the tablet. Some of the challenges I had were navigating through the tablet, making/accepting calls via } \\
\text { zoom, connecting the audio, and remembering how to synchronize the watch with the app." [Patient \#7] }\end{array}$ \\
\hline \multirow{2}{*}{$\begin{array}{l}\text { Recommendation to } \\
\text { offer the } \\
\text { teleprehabilitation } \\
\text { services to other patients }\end{array}$} & 3 & • "Keep going! You have to do this to get out of the hospital quickly" [Patient\#5] \\
\hline & & $\begin{array}{l}\text { •I am grateful to have had the support of the [prehabilitation] team during [neoadjuvant] treatments and before } \\
\text { surgery" [Patient \#7] }\end{array}$ \\
\hline
\end{tabular}

The Technologies

According to the patients, the median score for user-friendliness of the technological system was 90\% (range: 53 to $100 \%$ ). Three patients mentioned trouble using the tablet and the watch; see Table 6 for the nature of the patient's challenges with the technologies.

Table 6

Patients' Technological Challenges

\begin{tabular}{ll}
\hline The Tablet & $\begin{array}{l}\text { The Watch } \\
\text { - Mandatory software updates on the tablet }\end{array}$ \\
$\begin{array}{l}\text { - Difficulty connecting the watch to the tablet (through Bluetooth } \\
\text { connection) }\end{array}$ \\
$\begin{array}{l}\text { - Connecting the audio during the video-conferencing meeting } \\
\text { - Locating the messages sent by the prehabilitation healthcare } \\
\text { professionals. }\end{array}$
\end{tabular}

\section{Discussion}

The aims of this cohort study were to report the impact of delivery of a novel method of prehabilitation services to cancer patients and, more specifically, issues related to technology and patients' experience. The main results showed that telehealth interventions were well received by patients, allowing for greater flexibility in clinical scheduling and exercise interventions. However, challenges remain in its seamless implementation. Notably, hurdles to overcome include the adoption of the technologies by patients and the multidisciplinary team, the difficulty of acquiring accurate data on patient physical activity, and the initial costs of acquiring the new technologies. The patients' experience also highlighted two aspects of the program: the appreciation for services and the support received and the user-friendliness of the technological system provided.

\section{Addressing the literature gaps}

Telehealth has experienced rapid growth since the COVID-19 pandemic (6,9), enhancing its utility for diverse clinical applications. (17). Nevertheless, the literature is lacking with respect to the context of interventions and the clinical populations, more specifically with regard to acute care and elderly patients. Even further, there is a lack of consensus in the literature concerning the optimal technological systems for the successful delivery of teleprehabilitation (9). 
Two technological approaches have been proposed to overcome technology adoption barriers: 1. readily accessible technologies and 2. combining activity monitoring devices with a secure videoconferencing platform. In the context of the present study, most of the patients were older and had limited access to technologies and the internet.

\section{The Program}

Teleprehabilitation is a novel concept, even more so in high-risk surgical cancer patients, prompting the need for increased documentation of its implementation in clinical practice. Two other studies notably documented their delivery of teleprehabilitation programs in this population, both of which were pilot studies with bimodal (exercise and nutrition (18)) and unimodal (exercise-only (19)) interventions. The first study by Bruns et al. provided prehabilitation to frail elderly cancer surgery candidates through a home-based electronic prototype that was created solely for this purpose(18), while the other study by Piraux et al. used a virtual exercise prescription application and an exercise monitoring watch for esophageal cancer patients (19). The exercise interventions in both studies were significantly different (daily 7-minute prerecorded exercise videos and nutritional recommendations (18) vs three sessions weekly, including a 30-minute aerobic, a 30-minute resistance and an IMT training component (19)). Nonetheless, both studies included a weekly phone call to assess adherence (18) (19). Bruns et al. acknowledged that although self-reported adherence was high, the lack of supervision may lead to lower-quality execution and prevent individualization of interventions $(18,20)$. Furthermore, Piraux et al. identified that the application interface may not be suitable for patients with lower technological literacy. Nevertheless, patients reported a high overall level of satisfaction with the teleprehabilitation program, and the authors discussed the added value of reducing transportation burden on patients' schedules $(12,13,21)$.

In relation to the literature, the current findings reinforce the importance of the appropriate selection of technologies with regard to the simplicity of use (i.e., user-friendly interface) and the possibility of supervising interventions remotely (9). In all studies, patients were satisfied and comfortable with the technologies, with few patients experiencing minor technical difficulties. Distinctly, the current study included synchronous exercise counseling, which was mentioned to be a limitation of the Fit4SurgeryTV program (18). Increased supervision has been shown to yield greater adherence rates and improvements in health and functional outcomes (20). The latter may be attributed in part to increased attention from clinicians who can ensure that patients properly adhere to the exercises prescribed, attaining the appropriate intensity and duration, while applying the necessary modifications and progressions, which aligned with the findings of the current study.

Another novelty of the study was the acquisition of a large variety of exercise metrics from the physical activity watch. Prior to the study, most home-based interventions were reliant on patients' capacity to self-assess their compliance with the programs (22). Physical activity monitoring devices provide clinicians with important insight into physical activity levels, and unlike phone calls, they offer a more quantitative perspective of movement patterns and behaviours on a day-to-day basis. The technologies create a communication portal between patients and their clinicians, allowing them to better appreciate the patient's overall exercise volume (i.e., frequency, intensity, time, type). This increased access to information may be helpful in view of adapting and progressing the prescription throughout the preoperative period according to patients' capacities; however, it brings forth a new challenge for the scientific community to properly quantify and interpret adherence to the program.

\section{Patient's Experience}

The high satisfaction reported in the current study aligns with many telehealth-based interventions in the perioperative field $(13,18,19,21)$. Notably, a telerehabilitation by Kairy et al. aimed to document solely the patient's perspective of a telerehabilitation program after hip arthroplasty through interviews (13). In both studies, patients emphasized their appreciation for the technology's ease of use and the reduced need for hospital commutes. Moreover, patients in this retrospective cohort reported a sense of accomplishment in being able to positively impact their respective care trajectories.

\section{Limitations}

While this study showed great clinical potential for the implementation of teleprehabilitation, it is not without its limitations, notably related to its design, evaluations, and interventions. First, due to the design based on the need to describe the methodology, this is an exploratory study with a small cohort with diverse demographics, cancer types, pathologies, and disease management approaches. As such, the data documented in the functional and clinical trajectories section should be seen as contextual information, not presented to draw conclusions. Second, given that some patients were unable to visit the prehabilitation clinic, clinicians performed evaluations in surgical clinics or inpatient units. The latter leads to a minimal functional and demographic assessment, as the exercise physiologist cannot always access the material or space to conduct all tests. Furthermore, several patients did not attend follow-up health assessments due to concerns pertaining to commuting, COVID-19 or conflicts with other medical appointments. Last, a major limitation of the described services lies in the fact that videoconferencing interventions were conducted uniquely by exercise physiologists. Not all the members of the multidisciplinary team were trained or equipped to deliver their services using the new technologies. Future studies should investigate the feasibility and impact of videoconferencing multimodal interventions beyond the scope of exercise.

\section{Conclusion}

This study aimed to document the implementation process of teleprehabilitation in cancer patients after the first wave of the COVID-19 pandemic and report the successes and challenges of this new intervention modality. The successes identified were related to positive acceptance by patients of this technology, convenient clinic scheduling and interventions, and optimal medical follow-up. The challenges observed included the patients' learning curves, the limited acquisition of physical activity data, the adjustment of the multidisciplinary team to new technologies, the postoperative loss in follow-up, and the initial costs of the technologies. This study further aimed to document the patient's experience of receiving multimodal teleprehabilitation services. On this subject, patients were satisfied with the teleprehabilitation program (i.e., services delivered), and they perceived the technologies provided to be user-friendly. Future 
studies should also investigate the feasibility and validity of virtual functional health assessments in the case that visiting the hospital is not a viable option (23), in addition to investigating ways to improve the documentation of adherence to lifestyle interventions.

\section{Declarations}

\section{Funding}

Non-applicable due to the retrospective nature of the study

\section{Competing Interests}

The authors declare that they have no known competing financial interests or personal relationships that could have appeared to influence the work reported in this paper.

\section{Availability of data and materials}

The principal investigator (F.C.) and the research coordinator (B.T.) will have full access to the final database.

\section{Authors Contributions}

All authors contributed significantly to either the elaboration or writing and review of the trial, with K.D., G.L. and F.C. as the main contributors. B.T. assisted with the coordination of the system and study. All authors read and approved the manuscript.

\section{Ethics Approval and Consent to Participate}

Following referral by surgical investigators, the patients were contacted by a member of the research team who discussed the technology-assisted support and expectations of their involvement. If the patient expressed interest, they were invited to visit the prehabilitation clinic at the Montreal Genera Hospital where informed consent was provided.

\section{Consent for Publication}

Non-applicable due to the retrospective nature of the study

\section{References}

1. Schmunk R. Catching up on B.C. surgery backlog will take up to 2 years, province says. 2020 May 7;

2. Ross S. Montreal hospitals backtrack on plan to hire unskilled workers as operating-room help. CTV News Montreal Digital Reporter [Internet]. 2021 Apr 2; Available from: https://montreal.ctvnews.ca/mobile/montreal-hospitals-backtrack-on-plan-to-hire-unskilled-workers-as-operating-room-help1.5373151

3. Søgaard M, Thomsen RW, Bossen KS, Sørensen HT, Nørgaard M. The impact of comorbidity on cancer survival: A review. Clin Epidemiol. 2013;5(SUPP 1):3-29.

4. Silver JK. Prehabilitation could save lives in a pandemic [Internet]. Vol. 369, The BMJ. 2020. p. m1386. Available from: http://www.bmj.com/lookup/doi/10.1136/bmj.m1386

5. Sell NM, Silver JK, Rando S, Draviam AC, Santa Mina D, Qadan M, et al. Prehabilitation Telemedicine in Neoadjuvant Surgical Oncology Patients During the Novel COVID-19 Coronavirus Pandemic.

6. Silver JK. Prehabilitation May Help Mitigate an Increase in COVID-19 Peri-Pandemic Surgical Morbidity and Mortality. Am J Phys Med Rehabil. 2020 Apr; 1 .

7. Simcock R. Principles and guidance for prehabilitation within the management and support of people with cancer In partnership with Acknowledgements [Internet]. 2019. Available from: https://www.researchgate.net/publication/336617250

8. Awasthi R, Minnella EM, Ferreira V, Ramanakumar A V., Scheede-Bergdahl C, Carli F. Supervised exercise training with multimodal pre-habilitation leads to earlier functional recovery following colorectal cancer resection. Acta Anaesthesiol Scand. 2019;63(4):461-7.

9. Lambert G, Drummond K, Ferreira V, Carli F. Teleprehabilitation during COVID-19 pandemic: the essentials of "what" and "how." 2020;

10. Communication ZV. Zoom.us [Internet]. 2019 [cited 2020 May 8]. Available from: https://zoom.us/pricing

11. Electro Oy P. Polar Watch [Internet]. 2019 [cited 2020 May 8]. Available from: https://www.polar.com/ca-en

12. Lambert G, Alos N, Bernier P, Laverdière C, Drummond K, Dahan-Oliel N, et al. Patient and parent experiences with group telerehabilitation for child survivors of acute lymphoblastic leukemia. Int J Environ Res Public Health. 2021;18(7).

13. Kairy D, Tousignant M, Leclerc N, Côté AM, Levasseur M. The patient's perspective of in-home telerehabilitation physiotherapy services following total knee arthroplasty. Int J Environ Res Public Health. 2013;10(9):3998-4011.

14. Butland RJA, Pang J, Gross ER, Woodcock AA, Geddes DM. Two-, six-, and 12-minute walking testsinrespiratorydisease. 1982.

15. Seely AJE, Ivanovic J, Threader J, Al-Hussaini A, Al-Shehab D, Ramsay T, et al. Systematic classification of morbidity and mortality after thoracic surgery. Ann Thorac Surg. 2010;90(3):936-42. 
16. Ghasemi A, Zahediasl S. Normality tests for statistical analysis: A guide for non-statisticians. Int J Endocrinol Metab. 2012 Apr;10(2):486-9.

17. Horsley S, Schock G, Grona SL, Montieth K, Mowat B, Stasiuk K, et al. Use of real-time videoconferencing to deliver physical therapy services: A scoping review of published and emerging evidence. J Telemed Telecare. 2019;

18. Bruns ERJ, Argillander TE, Schuijt HJ, Van Duijvendijk P, Van Der Zaag ES, Wassenaar EB, et al. Fit4SurgeryTV At-home Prehabilitation for Frail Older Patients Planned for Colorectal Cancer Surgery: A Pilot Study. In: American Journal of Physical Medicine and Rehabilitation. Lippincott Williams and Wilkins; 2019. p. 399-406.

19. Piraux E, Caty G, Reychler G, Forget P, Deswysen Y. Feasibility and Preliminary Effectiveness of a Tele-Prehabilitation Program in Esophagogastric Cancer Patients. J Clin Med. 2020;9(7):2176.

20. Stout NL, Baima J, Swisher AK, Winters-Stone KM, Welsh J. A Systematic Review of Exercise Systematic Reviews in the Cancer Literature (2005-2017). PM R. 2017;9(9):S347-84.

21. Doiron-Cadrin P, Kairy D, Vendittoli PA, Lowry V, Poitras S, Desmeules F. Feasibility and preliminary effects of a tele-prehabilitation program and an inperson prehablitation program compared to usual care for total hip or knee arthroplasty candidates: a pilot randomized controlled trial. Disabil Rehabil. 2020 Mar 26;42(7):989-98.

22. Bollen JC, Dean SG, Siegert RJ, Howe TE, Goodwin VA. A systematic review of measures of self-reported adherence to unsupervised home-based rehabilitation exercise programmes, and their psychometric properties. BMJ Open. 2014;4(6).

23. Blair CK, Harding E, Herman C, Boyce T, Demark-Wahnefried W, Davis S, et al. Remote assessment of functional mobility and strength in older cancer survivors: Protocol for a validity and reliability study. Vol. 9, JMIR Research Protocols. JMIR Publications Inc.; 2020.

\section{Figures}

Figure 1. The Patient's Trajectory through the Teleprehabilitation Pathway

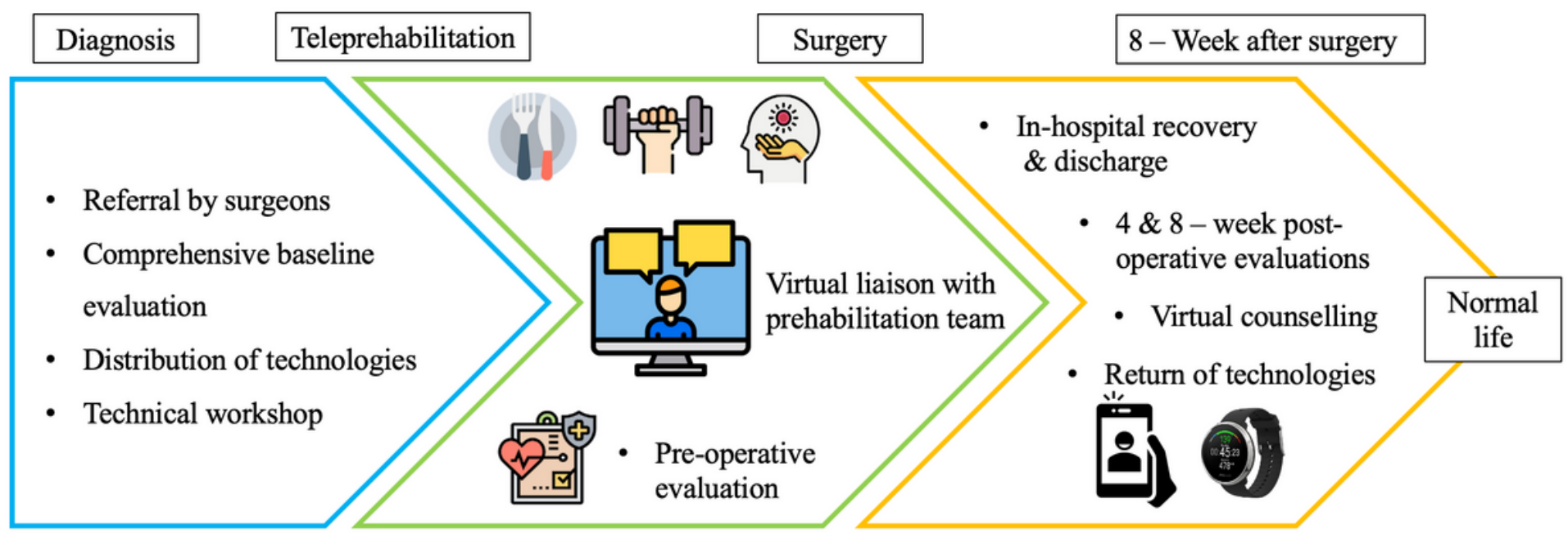

$<$ div $>$ Icons made by $<$ a href="https://www.flaticon.com/authors/xnimrodx" title="xnimrodx" $>$ xnimrodx $</ a>$ from $<$ a href="https://www.flaticon.com/" title="Flaticon" $>$ www.flaticon.com</a $></$ div $>$; $<$ div $>$ Icons made by $<$ a href="https://www.flaticon.com/authors/pixel-perfect" title="Pixel perfect" $>$ Pixel perfect $</ a>$ from $<$ a href="https $: /$ www.flaticon.com/" title="Flaticon" $>$ www.flaticon.com $</$ a $></$ div $>$

Figure 1

"See image above for figure legend"

\section{Supplementary Files}

This is a list of supplementary files associated with this preprint. Click to download.

- AppendixASatisfactionquestionnaire.docx 